\title{
Parametric global optimisation for bilevel programming
}

\author{
Nuno P. Faísca • Vivek Dua • Berç Rustem • \\ Pedro M. Saraiva • Efstratios N. Pistikopoulos
}

Received: 20 September 2006 / Accepted: 20 September 2006 / Published online: 27 October 2006

(C) Springer Science+Business Media B.V. 2006

\begin{abstract}
We propose a global optimisation approach for the solution of various classes of bilevel programming problems (BLPP) based on recently developed parametric programming algorithms. We first describe how we can recast and solve the inner (follower's) problem of the bilevel formulation as a multi-parametric programming problem, with parameters being the (unknown) variables of the outer (leader's) problem. By inserting the obtained rational reaction sets in the upper level problem the overall problem is transformed into a set of independent quadratic, linear or mixed integer linear programming problems, which can be solved to global optimality. In particular, we solve bilevel quadratic and bilevel mixed integer linear problems, with or without right-hand-side uncertainty. A number of examples are presented to illustrate the steps and details of the proposed global optimisation strategy.
\end{abstract}

Keywords Bilevel programming · Parametric programming · Quadratic . Mixed-integer . Uncertainty

N. P. Faísca · B. Rustem · E. N. Pistikopoulos $(\varangle)$

Centre for Process Systems Engineering, Imperial College London, London SW7 2AZ, UK

e-mail: e.pistikopoulos@imperial.ac.uk

V. Dua

Centre for Process Systems Engineering, University College London, London WC1E 7JE, UK

e-mail: v.dua@ucl.ac.uk

P. M. Saraiva

Gepsi- PSE Group, Department of Chemical Engineering, University of Coimbra,

Coimbra 3030-290, Portugal

e-mail: pas@eq.uc.pt

N. P. Faísca

e-mail: n.faisca@imperial.ac.uk

B. Rustem

e-mail: br@doc.ic.ac.uk 


\section{Introduction}

Multilevel optimisation problems have attracted considerable attention from the scientific and economic community in recent years. Due to its many applications, multilevel and in particular bilevel programming have evolved significantly. Bilevel programming problems (BLPP) involve a hierarchy of two optimisation problems, of the following form (Vicente and Calamai 1994a; Floudas 2000; Dempe 2003):

$$
\begin{array}{ll} 
& \min _{x, y} F(x, y), \\
\text { s.t. } & G(x, y) \leq 0, \\
& x \in X, \\
y & \in \operatorname{argmin}\{f(x, y): g(x, y) \leq 0, y \in Y\}
\end{array}
$$

where $X \subseteq \mathbb{R}^{n x}$ and $Y \subseteq \mathbb{R}^{n y}$ are both compact convex sets; $F$ and $f$ are real functions: $\mathbb{R}^{(n x+n y)} \rightarrow \mathbb{R} ; \mathrm{G}$ and $g$ are vectorial real functions, $G: \mathbb{R}^{(n x+n y)} \rightarrow \mathbb{R}^{n u}$ and $g: \mathbb{R}^{(n x+n y)} \rightarrow \mathbb{R}^{n l} ; n x, n y \in \mathbb{N}$ and $n u, n l \in \mathbb{N} \cup\{0\}$. The following definitions are associated to Problem (1):

Relaxed feasible set (or constrained region),

$$
\Omega=\{x \in X, y \in Y: G(x, y) \leq 0, g(x, y) \leq 0\} .
$$

Lower level feasible set,

$$
\mathrm{C}(x)=\{y \in Y: g(x, y) \leq 0\} .
$$

Follower's rational reaction set,

$$
\mathrm{M}(x)=\{y \in Y: y \in \operatorname{argmin}\{f(x, y): y \in \mathrm{C}(x)\}\} .
$$

Inducible region,

$$
\mathrm{IR}=\{x \in X, y \in Y:(x, y) \in \Omega, y \in \mathrm{M}(x)\} .
$$

Applications of bilevel and multilevel programming include design optimisation problems in process systems engineering (Clark 1990; Clark and Westerberg 1990); design of transportation networks (LeBlanc and Boyce 1985); agricultural planning (Fortuny-Amat and McCarl 1981); management of multi-divisional firms (Ryu et al. 2004) and hierarchical decision-making structures (Fortuny-Amat and McCarl 1981). These multilevel problems are classified accordingly to the number of levels and the type of their cost functions and variables: if the problem has two levels, where both cost functions are affine functions and the variables are continuous, the problem is classified as a linear BLPP; if at least one of these functions has a quadratic expression, it is a quadratic BLPP; adding uncertainty to the formulations results in a BLPP with uncertainty; on the other hand, if binary and continuous variables coexist in the same bilevel problem formulation, it corresponds to a mixed integer BLPP.

Recently, Pistikopoulos and co-workers (Dua and Pistikopoulos 2000, Dua et al. 2002) have proposed novel solution algorithms which open the possibility of using a general framework to address general classes of bilevel and multilevel programming problems. These algorithms are based on parametric programming theory (Acevedo and Pistikopoulos 1997, Dua 2000) and use of the Basic Sensitivity Theorem (Fiacco 1976, 1983). This approach can be classified as a Reformulation Technique 
(Visweswaran et al. 1996) since the bilevel problem is transformed into a number of quadratic or linear problems. The main idea is to divide the follower's feasible area into different rational reaction sets, and search for the global optimum of a simple quadratic (or linear) programming problem in each area.

\subsection{Global optimum of a bilevel programming problem}

While for an optimal control problem (one-player problem) there is a well-defined concept for optimality, the same is not always true for multi-person games (Başar and Olsder 1982).

In the case of bilevel programming, Vicente (1992), Visweswaran et al. (1996), Shimizu et al. (1997), Floudas et al. (1999), Floudas (2000) and Dempe et al. (2005) interpret the optimisation problem as a leader's problem, F, and search for the global minimum of $F$. The solution point obtained for the follower's problem, $f$, will respect the stationary (KKT) conditions and hence it can be any stationary point.

Obviously, this solution strategy is acceptable when the player in the upper level of the hierarchy is in the most "powerful" position, and the other levels just react to the decision of their leader. Such approach is sensible in many engineering applications of bilevel programming (for instance, see Clark and Westerberg 1990; Clark 1990). It is also a valid strategy for the cases of decentralised manufacturing and financial structures, when the leader has a full insight and control of the overall objectives and strategy of the corporation, while the follower does not.

However, this is not always the case. For example, using the feedback Stackelberg solution, where at every level of play a Stackelberg equilibrium point is searched, the commitment of the leader for his/her decision increases with the number of players involved. Cao and Chen (2006) present an example where the sacrifice of the leader's objective on behalf of the followers results in a better solution for both levels. Similar solution strategies have also been studied (Tabucanon 1988; Lai 1996; Shih et al. 1996; Cao and Chen 2006).

Theorem 1 (Vicente 1992) If for each $x \in X$, f and $g$ are twice continuously differentiable functions for every $y \in C(x)$, f is strictly convex for every $y \in C(x)$ and $C(x)$ is a convex and compact set, then $M(\cdot)$ is a real-valued function, continuous and closed.

If Theorem 1 applies and assuming that $\mathrm{M}(x)$ is non-empty, then $\mathrm{M}(x)$ will have only one element, which is $y(x)$. Thus, Eq. (1) can be reformulated as:

$$
\begin{array}{ll} 
& \min _{x, y} F(x, y(x)), \\
\text { s.t. } & G(x, y(x)) \leq 0, \\
& x \in C_{r f}, \\
& C_{r f}=\{x \in X: \exists y \in Y, g(x, y) \leq 0\} .
\end{array}
$$

Considering that $f$ is a convex real function, the function $y(x)$ can be computed as a linear conditional function based on parametric programming theory, as follows (Dua et al. 2002): 


$$
y(x)=\left\{\begin{array}{lll}
m^{1}+n^{1} x, & \text { if } & H^{1} x \leq h^{1}, \\
m^{2}+n^{2} x, & \text { if } & H^{2} x \leq h^{2}, \\
\vdots & & \\
m^{k}+n^{k} x, & \text { if } & H^{k} x \leq h^{k}, \\
\vdots & & \\
m^{K}+n^{K} x, & \text { if } & H^{K} x \leq h^{K},
\end{array}\right.
$$

where, $n^{k}, m^{k}$ and $h^{k}$ are real vectors and $H^{k}$ is a real matrix.

Theorem 2 (Vicente 1992) If the assumptions of Theorem 1 hold, $F$ is a real continuous function, $X$ and the set defined by $G(x, y)$ are compact, and if $\{\exists x \in X: G(x, y(x)) \leq 0\}$, then there is a global solution for Problem (1).

Since an explicit expression for $y$ can be computed, if the assumptions of Theorem 2 hold, and the two players have convex functions to optimise, then the global optimum for Problem (1) can be obtained via the parametric programming approach.

The advantage of using this approach is that the final solution will consider the possibility of existence of other global minima, which could correspond to better solutions for the follower. Moreover, the parametric nature of the leader's problem is preserved.

Regarding computational complexity, a number of authors have shown that BLPP are $\mathcal{N} \mathcal{P}$-Hard (Hansen et al. 1992, Deng 1998). Furthermore, Vicente et al. (1994b) proved that even checking for a local optimum is a $\mathcal{N} \mathcal{P}$-Hard problem.

The objective of this work is to describe a parametric programming framework which can solve different classes of multilevel programming problems to global optimality. Section 2 presents the fundamental developments for the quadratic bilevel programming case. The theory is extended, in Sect. 3, to cover the existence of RHS uncertainty, and Sect. 4 addresses mixed integer bilevel programming.

\section{Quadratic bilevel programming}

Consider the following general quadratic BLPP:

$$
\begin{aligned}
& \quad \min _{x, y} F(x, y)=L_{1}+L_{2} x+L_{3} y+\frac{1}{2} x^{T} L_{4} x+y^{T} L_{5} x+\frac{1}{2} y^{T} L_{6} y, \\
& \text { s.t. } G_{1} x+G_{2} y+G_{3} \leq 0, \\
& \quad \min _{y} f(x, y)=l_{1}+l_{2} x+l_{3} y+\frac{1}{2} x^{T} l_{4} x+y^{T} l_{5} x+\frac{1}{2} y^{T} l_{6} y, \\
& \text { s.t. } \quad g_{1} x+g_{2} y+g_{3} \leq 0,
\end{aligned}
$$

where $x$ and $y$ are the optimisation variables, $x \in X \subseteq \mathbb{R}^{n x}$ and $y \in Y \subseteq \mathbb{R}^{n y}$. $\left[L_{2}\right]_{1 \times n x}$, $\left[L_{3}\right]_{1 \times n y},\left[L_{4}\right]_{n x \times n x},\left[L_{5}\right]_{n y \times n x},\left[L_{6}\right]_{n y \times n y},\left[l_{2}\right]_{1 \times n x},\left[l_{3}\right]_{1 \times n y},\left[l_{4}\right]_{n x \times n x},\left[l_{5}\right]_{n y \times n x}$ and $\left[l_{6}\right]_{n y \times n y}$ are matrices defined in the real space. The matrices $\left[G_{1}\right]_{n u \times n x},\left[G_{2}\right]_{n u \times n y}$, $\left[G_{3}\right]_{n u \times 1},\left[g_{1}\right]_{n u \times n x},\left[g_{2}\right]_{n u \times n y},\left[g_{3}\right]_{n u \times 1}$ correspond to the constraints, also defined in the real space.

Focusing the attention on the follower's optimisation problem, considering $x$ as a parameter vector and operating a variable change $\left(z=y+l_{6}^{-1} l_{5} x\right)$, it can be rewritten as the following multiparametric quadratic programming (mp-QP) problem: 


$$
\begin{aligned}
& \min _{z} f^{\prime}(x, z)=l_{1}^{\prime}+l_{2}^{\prime} x+\frac{1}{2} x^{T} l_{4}^{\prime} x+\left\{l_{3}^{\prime} z+\frac{1}{2} z^{T} l_{6}^{\prime} z\right\}, \\
& \text { s.t. } g_{2}^{\prime} z \leq g_{3}^{\prime}+g_{1}^{\prime} x,
\end{aligned}
$$

where: $l_{1}^{\prime}=l_{1} ; l_{2}^{\prime}=l_{2}-l_{3} l_{6}^{-1} l_{5} ; l_{3}^{\prime}=l_{3} ; l_{4}^{\prime}=l_{4}-l_{5}^{T} l_{6}^{-1} l_{5} ; l_{5}^{\prime}=0 ; l_{6}^{\prime}=l_{6} ; g_{1}^{\prime}=$ $-\left(g_{1}-g_{2} l_{6}^{-1} l_{5}\right) ; g_{2}^{\prime}=g_{2} ; g_{3}^{\prime}=-g_{3}$.

The mp-QP Problem (9) can be solved applying the algorithm of Dua et al. (2002) (Appendix A). As a result, a set of rational reaction sets (Definition 4) is obtained for different regions of $x$ :

$$
z^{k}=m^{k}+n^{k} x ; \quad H^{k} x \leq h^{k}, \quad k=1,2, \ldots, K .
$$

By incorporating the expressions (10) into Problem (8) results in the the following $K$ quadratic problems:

$$
\begin{aligned}
& \quad \min _{x} F^{\prime}(x)=L_{1}^{\prime k}+L_{2}^{\prime k} x+\frac{1}{2} x^{T} L_{4}^{\prime k} x, \\
& \text { s.t. } G_{1}^{\prime k} x \leq G_{3}^{\prime k}
\end{aligned}
$$

with:

$$
\begin{aligned}
& L_{1}^{\prime k}=L_{1}+L_{3} m^{k}+\frac{1}{2} m^{k^{T}} L_{6} m^{k}, \\
& L_{2}^{\prime k}=L_{2}+L_{3} n^{k}-L_{3} l_{6}^{-1} l_{5}+m^{k^{T}} L_{5}+m^{k^{T}} L_{6} n^{k}-m^{k^{T}} L_{6} l_{6}^{-1} l_{5}, \\
& L_{4}^{\prime k}=L_{4}+2 n^{k} L_{5}-2 l_{5}^{T} l_{6}^{-1} L_{5}+n^{k^{T}} L_{6} n^{k}-2 n^{k^{T}} L_{6} l_{6}^{-1} l_{5}+l_{5}^{T} l_{6}^{-1} L_{6} l_{6}^{-1} l_{5}, \\
& G_{1}^{\prime}=G_{1}+G_{2} n^{k}-G_{2} l_{6}^{-1} l_{5}, \\
& G_{3}^{\prime}=-\left(G_{3}+G_{2} m^{k}\right), \\
& G_{1}^{\prime k}=\left[G_{1}^{\prime} \mid H^{k}\right]_{(n x) \times\left(n u+n_{h^{k}}\right)}^{T}, \\
& G_{3}^{\prime k}=\left[G_{3}^{\prime} \mid h^{k}\right]_{(1) \times\left(n u+n_{h^{k}}\right)}^{T} .
\end{aligned}
$$

Clearly, the solution of the BLLP Problem (8) is the minimum along the $K$ solutions of Problem (11).

Remark 1 The artificial variable, $z$, introduced in Problem (9) is only necessary if $l_{5} \neq 0$. In all other cases the multi-parametric problem can be easily formulated through algebraic manipulations.

Remark 2 When one of the matrices $l_{6}^{\prime}, L_{4}^{\prime k}$ is null the optimisation problem where these are involved becomes linear. Particulary, if $l_{6}^{\prime}=\underline{0}$, Problem (9) is transformed into a mp-LP; on the other hand, if $L_{4}^{\prime k}=\underline{0}$, Problem (11) becomes a LP problem. In both cases, the solution procedure is not affected, due to the fact that the Basic Sensitivity Theorem (Fiacco 1976, 1983) also applies to the mp-LP problem.

Remark 3 The expression for the artificial variable introduced, $z$, is only valid when $l_{6}$ is symmetric. If not, with the following transformation:

$$
\bar{l}_{6}=\left\{\frac{l_{6}+l_{6}^{T}}{2}\right\}
$$


the resulting matrix is non-singular. If the resulting matrix is singular the expression for the artificial variable should be given by:

$$
\begin{aligned}
& z=y+A x, \\
& \quad \text { where } A \text { should satisfy: }\left\{A \in \mathbb{R}^{n x \times n x}: l_{5}-\left(\frac{1}{2} l_{6}+\frac{1}{2} l_{6}^{T}\right) A=0\right\} .
\end{aligned}
$$

In this case, several solutions for the system above can exist. However, as long as the bilinear terms are eliminated in Problem (9) any solution can be selected.

Remark 4 This technique is not valid when at the same time: (1) $f$ is a pure quadratic cost function; (2) $f$ involves bilinear terms and (3) matrix $\bar{l}_{6}$ is singular.

Observing Formulation (11) we can conclude that the parametric programming approach (Table 1), transforms the original quadratic BLPP into simple quadratic problems, for which a global optimum can be reached.

In the following subsections, examples are presented for LP $|\mathrm{LP}, \mathrm{LP}| \mathrm{QP}$ and $\mathrm{QP} \mid \mathrm{QP}$ BLPP.

2.1 LP|LP bilevel programming problem

Consider the following linear BLPP (Bard and Falk 1982):

$$
\begin{array}{rl}
\quad \min _{x, y} & F(x, y)=-8 x_{1}-4 x_{2}+4 y_{1}-40 y_{2}+4 y_{3}, \\
\text { s.t. } \min _{y} & f(x, y)=x_{1}+2 x_{2}+y_{1}+y_{2}+2 y_{3}, \\
\text { s.t. } & -y_{1}+y_{2}+y_{3} \leq 1, \\
& 2 x_{1}-y_{1}+2 y_{2}-0.5 y_{3} \leq 1, \\
& 2 x_{2}+2 y_{1}-y_{2}-0.5 y_{3} \leq 1, \\
& y \geq 0 \\
& x \geq 0 .
\end{array}
$$

Problem (12) was solved using the steps described in Table (1):

Step 1 Formulate a mp-LP problem for the lower level:

$$
\begin{array}{ll} 
& \min _{y} f(x, y)=x_{1}+2 x_{2}+y_{1}+y_{2}+2 y_{3}, \\
\text { s.t. } & -y_{1}+y_{2}+y_{3} \leq 1 \\
& -y_{1}+2 y_{2}-0.5 y_{3} \leq 1-2 x_{1}, \\
& 2 y_{1}-y_{2}-0.5 y_{3} \leq 1-2 x_{2}, \\
& y \geq 0 \\
& x \geq 0 .
\end{array}
$$

Table 1 Parametric programming approach for a BLPP

\begin{tabular}{ll}
\hline Step & Description \\
\hline 1 & $\begin{array}{l}\text { Recast the inner problem as a multi-parametric } \\
\text { programming problem, with the leader's variables } \\
\text { being the parameters }(9)\end{array}$ \\
2 & $\begin{array}{l}\text { Solve the resulting problem using the suitable } \\
\text { multi-parametric programming algorithm }\end{array}$ \\
& $\begin{array}{l}\text { Substitute each of the } K \text { solutions in the leader's } \\
\text { problem, and formulate the } K \text { one level } \\
\text { optimisation problems }\end{array}$ \\
& $\begin{array}{l}\text { Compare the } K \text { optimum points and select the best one } \\
4\end{array}$
\end{tabular}


Step 2 The application of the mp-LP algorithm to the lower level results in the following five rational reaction sets in Table 2.

Step 3 Substituting each of the sets obtained into the leader's problem, five linear programming problems result (Table 3 ).

Step 4 Observing the best values achieved for each region (Table 3), the global solution is obtained for: $x_{1}=0 ; x_{2}=0.9 ; y_{1}=0 ; y_{2}=0.6 ; y_{3}=0.4(F=-26 ; f=3.2)$.

The global minimum obtained is the same as the one reported by Floudas et al. (1999). Here only the solution of a single mp-LP and five LP was required to obtain the global minimum; whereas for the same problem Shimizu et al. (1997) report that their strategy requires the solution of ten subproblems. Clearly, the computational efficiency of the proposed procedure depends on the performance of the underlying multi-parametric programming algorithm (which, in independent studies, has been reported as robust and efficient: Dua and Pistikopoulos 2000; Dua et al. 2002; Sakizlis et al. 2003; Sakizlis et al. 2004 a, b).

\subsection{LP $\mid \mathrm{QP}$ bilevel programming problem}

Consider a linear cost function at the leader's level and a quadratic at the lower level:

$$
\begin{array}{rl}
\min _{x, y} & F(x, y)=2 x_{1}+2 x_{2}-3 y_{1}-3 y_{2}-60, \\
\text { s.t. } x_{1}+x_{2}+y_{1}-2 y_{2}-40 \leq 0, & \\
\min _{y} & f(x, y)=\left(y_{1}-x_{1}+20\right)^{2}+\left(y_{2}-x_{2}+20\right)^{2}, \\
\text { s.t. } \quad & -x_{1}+2 y_{1} \leq-10, \\
& -x_{2}+2 y_{2} \leq-10, \\
& 0 \leq x \leq 50 \\
& -10 \leq y \leq 20 .
\end{array}
$$

\begin{tabular}{|c|c|c|}
\hline$k$ & $y^{k}(x)=m^{k}+\left(n_{k}-l_{6}^{-1} l_{5}\right) x$ & $H^{k} x \leq h_{i}^{k}$ \\
\hline 1 & $\begin{array}{l}y_{1}(x)=0 \\
y_{2}(x)=0 \\
y_{3}(x)=0\end{array}$ & $0 \leq x \leq \frac{1}{2}$ \\
\hline 2 & $\begin{array}{l}y_{1}(x)=-1+2 x_{1} \\
y_{2}(x)=0\end{array}$ & $\begin{array}{l}0 \leq x_{2} \\
4 x_{1}+2 x_{2} \leq 3\end{array}$ \\
\hline 3 & $\begin{array}{l}y_{3}(x)=0 \\
y_{1}(x)=\frac{2}{3} x_{1}-\frac{2}{3} x_{2} \\
y_{2}(x)=0 \\
y_{3}(x)=-2+\frac{8}{3} x_{1}+\frac{4}{3} x_{2}\end{array}$ & $\begin{array}{l}\frac{1}{2} \leq x_{1} \\
0 \leq x_{2} \\
2 x_{1}+2 x_{2} \leq 3 \\
-x_{1}+x_{2} \leq 0 \\
-4 x_{1}-2 x_{2} \leq-3\end{array}$ \\
\hline 4 & $\begin{array}{l}y_{1}(x)=0 \\
y_{2}(x)=-\frac{2}{3} x_{1}+\frac{2}{3} x_{2} \\
y_{3}(x)=-2+\frac{4}{3} x_{1}+\frac{8}{3} x_{2}\end{array}$ & $\begin{array}{l}\frac{2}{3} x_{1}+\frac{10}{3} x_{2} \leq 3 \\
x_{1}-x_{2} \leq 0 \\
0 \leq x_{1} \\
-2 x_{1}-4 x_{2} \leq-3\end{array}$ \\
\hline 5 & $\begin{array}{l}y_{1}(x)=0 \\
y_{2}(x)=-1+2 x_{2} \\
y_{3}(x)=0\end{array}$ & $\begin{array}{l}0 \leq x_{1} \\
2 x_{1}+4 x_{2} \leq 3 \\
\frac{1}{2} \leq x_{2}\end{array}$ \\
\hline
\end{tabular}

Table 2 Rational reaction sets (Step 2) 
Table 3 Formulation of new problems (Steps 3 and 4)

\begin{tabular}{|c|c|c|c|}
\hline$k$ & Optimisation problem & Optimised variables & Function values \\
\hline 1 & $\begin{aligned} \min _{x} F & =-8 x_{1}-4 x_{2} \\
\text { s.t. } & 0 \leq x \leq \frac{1}{2}\end{aligned}$ & $\begin{array}{l}x_{1}=0.5, \quad x_{2}=0.5 \\
y_{1}=0, \quad y_{2}=0, \quad y_{3}=0\end{array}$ & $\begin{array}{l}F=-6 \\
f=1.5\end{array}$ \\
\hline 2 & $\begin{array}{ll} & \min _{x} F=-4 x_{2}-4 \\
\text { s.t. } & 0 \leq x_{2} \\
& 4 x_{1}+2 x_{2} \leq 3 \\
& 0.5 \leq x_{1}\end{array}$ & $\begin{array}{l}x_{1}=0.5, \quad x_{2}=0.5 \\
y_{1}=0, \quad y_{2}=0, \quad y_{3}=0\end{array}$ & $\begin{array}{l}F=-6 \\
f=1.5\end{array}$ \\
\hline 3 & $\begin{array}{ll} & \min _{x} F=\frac{16}{3} x_{1}-\frac{4}{3} x_{2}-4 \\
\text { s.t. } & 0 \leq x_{2} \\
& 2 x_{1}+2 x_{2} \leq 3 \\
& -x_{1}+x_{2} \leq 0 \\
& -4 x_{1}-2 x_{2} \leq-3\end{array}$ & $\begin{array}{l}x_{1}=0.5, \quad x_{2}=0.5 \\
y_{1}=0, \quad y_{2}=0, \quad y_{3}=0\end{array}$ & $\begin{array}{l}F=-6 \\
f=1.5\end{array}$ \\
\hline 4 & $\begin{array}{ll} & \min _{x} F=24 x_{1}-20 x_{2}-8 \\
\text { s.t. } & \frac{2}{3} x_{1}+\frac{10}{3} x_{2} \leq 3 \\
& x_{1}-x_{2} \leq 3 \\
& x_{1}-x_{2} \leq 0 \\
& 0 \leq x_{1} \\
& -2 x_{1}-4 x_{2} \leq-3\end{array}$ & $\begin{array}{ll}x_{1}=0, & x_{2}=0.9 \\
y_{1}=0, & y_{2}=0.6, \quad y_{3}=0.4\end{array}$ & $\begin{array}{l}F=-26 \\
f=3.2\end{array}$ \\
\hline 5 & $\begin{array}{ll} & \min _{x} F=-8 x_{1}-84 x_{2}+40 \\
\text { s.t. } & 0 \leq x_{1} \\
& 2 x_{1}+4 x_{2} \leq 3 \\
& 0.5 \leq x_{2}\end{array}$ & $\begin{array}{ll}x_{1}=0, & x_{2}=0.75 \\
y_{1}=0, & y_{2}=0.5, \quad y_{3}=0\end{array}$ & $\begin{array}{l}F=-23 \\
f=2\end{array}$ \\
\hline
\end{tabular}

The solutions found for this problem (Solutions 1 and 2) are compared to solutions reported in the literature (Aiyoshi and Shimizu 1981; Visweswaran et al. 1996), as shown in Table 4.

It is interesting to note: (1) Solutions 1 and 2 have the same (global) solution for the leader's problem, $F=0$. However, they differ in the solution of the follower's problem, (2) Solution 2 is identical to the solution reported in Visweswaran et al. (1996), (3) Solution 1 is the global solution (as discussed in 1.1), where both the leader and follower's cost functions are optimised. Thus, this comparison enhances a singular property of the framework developed in this work, which is the lower level optimisation; whereas most of the Reformulation Techniques just satisfy the requirement of having a stationary point, KKT optimality conditions, for the lower level, with this approach the decision maker optimises firstly the leader cost function but has the opportunity to optimise the lower level as well.

\subsection{QP $\mid \mathrm{QP}$ bilevel programming problem}

Consider the following problem, introduced by Muu and Quy (2003), which has quadratic functions in both levels:

Table 4 Different solutions for Problem (14)

\begin{tabular}{lccrrrr}
\hline Solution & $F$ & $f$ & $x_{1}$ & $x_{2}$ & $y_{1}$ & $y_{2}$ \\
\hline Aiyoshi and Shimizu (1981) & 5 & 100 & 25 & 30 & 5 & 10 \\
Visweswaran et al. (1996) & 0 & 200 & 0 & 0 & -10 & -10 \\
Solution 1 & 0 & 100 & 0 & 30 & -10 & 10 \\
Solution 2 & 0 & 200 & 0 & 0 & -10 & -10 \\
\hline
\end{tabular}




$$
\begin{aligned}
& \quad \min _{x, y} F(x, y)=y_{1}^{2}+y_{3}^{2}-y_{1} y_{3}-4 y_{2}-7 x_{1}+4 x_{2}, \\
& \text { s.t. } x_{1}+x_{2} \leq 1, \\
& \begin{array}{l}
\min _{y} f(x, y)=y_{1}^{2}+\frac{1}{2} y_{2}^{2}+\frac{1}{2} y_{3}^{2}+y_{1} y_{2}+\left(1-3 x_{1}\right) y_{1}+\left(1+x_{2}\right) y_{2}, \\
\text { s.t. } \quad 2 y_{1}+y_{2}-y_{3}+x_{1}-2 x_{2}+2 \leq 0, \\
\quad x \geq 0, \\
\quad y \geq 0 .
\end{array}
\end{aligned}
$$

Muu and Quy (2003) have solved this oligopolistic market example to find an $\epsilon$-global minimum $(\epsilon=0.01)$. The global minimum computed in the present work is compared to the former and presented in Table 5 .

It is interesting to note here that: (1) the solution obtained is in full agreement with the one reported in Muu and Quy (2003); (2) the solution of one mp-QP and one QP were required to arrive at the global solution.

\section{Bilevel programming with uncertainty}

Evans (1984) highlighted the importance of considering uncertainty/risk (e.g. prices, technological attributes, etc.) in the solution of decentralised decision makers. The presence of uncertainty in bilevel problems has been addressed before for the linear case (Ryu et al. 2004). Uncertainty is considered unstructured, taking any value between its bounds. In the present work we present an extension of our earlier work to the quadratic case.

We address the following quadratic BLPP with uncertainty, $\theta$ :

$$
\begin{aligned}
& \quad \min _{x, y} F(x, y, \theta)=L_{1}+L_{2} x+L_{3} y+\frac{1}{2} x^{T} L_{4} x+y^{T} L_{5} x+\frac{1}{2} y^{T} L_{6} y, \\
& \text { s.t. } G_{1} x+G_{2} y+G_{3} \leq G_{4} \theta, \\
& \quad \min _{y} f(x, y, \theta)=l_{1}+l_{2} x+l_{3} y+\frac{1}{2} x^{T} l_{4} x+y^{T} l_{5} x+\frac{1}{2} y^{T} l_{6} y, \\
& \text { s.t. } \quad g_{1} x+g_{2} y+g_{3} \leq g_{4} \theta,
\end{aligned}
$$

The steps for solving (16) are as follows:

1. Recast the inner problem as a mp-QP, with parameters being both $x$ and $\theta$. The solution obtained is similar to (10):

$$
z^{k}=m^{k}+n_{b}^{k} x+\bar{n}_{c}^{k} \theta, \quad H^{k} x+\bar{H}^{k} \theta \leq h^{k}, \quad k=1,2, \ldots, K .
$$

2. Incorporate expressions (17) in (16) to formulate $K \mathrm{mp}-\mathrm{QPs}$, with parameters being the uncertainty $\theta$ :

$$
\begin{aligned}
& \min _{x} F^{\prime}(x, \theta)=\bar{L}_{1}^{\prime k}+\bar{L}_{2}^{\prime k} x+\frac{1}{2} x^{T} \bar{L}_{4}^{\prime k} x, \\
& \text { s.t. } \bar{G}_{1}^{\prime k} x \leq \bar{G}_{3}^{\prime k}+\bar{G}_{4}^{\prime k} \theta,
\end{aligned}
$$

Table 5 Different solutions for Problem (15)

\begin{tabular}{llllllll}
\hline Solution & $F$ & $f$ & $x_{1}$ & $x_{2}$ & $y_{1}$ & $y_{2}$ & $y_{3}$ \\
\hline Muu and Quy (2003) & 0.6426 & 1.671 & 0.609 & 0.391 & 0 & 0 & 1.828 \\
Solution 1 & 0.6384 & 1.6799 & 0.6111 & 0.3889 & 0 & 0 & 1.8333 \\
\hline
\end{tabular}


where $\bar{L}_{1}^{\prime k}, \bar{L}_{2}^{\prime k}, \bar{L}_{4}^{\prime k}, \bar{G}_{1}^{\prime k}, \bar{G}_{2}^{\prime k}, \bar{G}_{4}^{\prime k}$ are appropriate matrices derived by algebraic manipulations.

We will illustrate the proposed procedure by revisiting example (15) with the addition of two uncertain parameters $\left(\theta_{1}, \theta_{2}\right)$ as follows:

$$
\begin{aligned}
& \quad \min _{x, y} F(x, y)=y_{1}^{2}+y_{3}^{2}-y_{1} y_{3}-4 y_{2}-7 x_{1}+4 x_{2}, \\
& \text { s.t. } x_{1}+x_{2} \leq 1+\theta_{1}, \\
& \begin{array}{rl}
\min _{y} & f(x, y)=y_{1}^{2}+\frac{1}{2} y_{2}^{2}+\frac{1}{2} y_{3}^{2}+y_{1} y_{2}+\left(1-3 x_{1}\right) y_{1}+\left(1+x_{2}\right) y_{2}, \\
\text { s.t. } \quad & 2 y_{1}+y_{2}-y_{3}+x_{1}-2 x_{2}+2 \leq \theta_{2}, \\
& x \geq 0 \\
& y \geq 0 \\
& \leq \theta_{1} \leq 0.25, \\
& \leq \theta_{2} \leq 0.5 .
\end{array}
\end{aligned}
$$

The solution of the inner mp-QP problem of Step 1 results in a single critical region, with the following parametric expressions:

$$
\begin{aligned}
& y_{1}=0, \\
& y_{2}=0, \\
& y_{3}=x_{1}-2 x_{2}-\theta_{2}+2, \\
& x_{1}+x_{2} \leq 1+\theta_{1}, \\
& -x_{1}+2 x_{2} \leq 2-\theta_{2}, \\
& x \geq 0 \\
& 0 \leq \theta_{1} \leq 0.25, \\
& 0 \leq \theta_{2} \leq 0.5
\end{aligned}
$$

Then, Step 2 involves (1) the substitution of the expressions in (20) into the leader's problem and (2) formulation and solution of the outer mp-QP problem, based on which the following results were obtained:

$$
\begin{aligned}
& x_{1}=0.444 \theta_{1}+0.556 \theta_{2}+0.611, \\
& x_{2}=0.556 \theta_{1}-0.556 \theta_{2}+0.389, \\
& 0 \leq \theta_{1} \leq 0.25, \\
& 0 \leq \theta_{2} \leq 0.5
\end{aligned}
$$

For the limiting case, when $\theta_{1}=0$ and $\theta_{2}=0$, the results obtained in (21) correspond to the results obtained in Sect. 2.3, Table 5.

In this example, Step 1 results in a single critical region (20). However, it is possible that, by the end, different parametric expressions are computed to the same critical region. We overcome this redundancy by keeping the best solution and discarding the others through the formal comparison procedure proposed by Acevedo and Pistikopoulos (1997).

\section{Mixed integer bilevel programming}

In many real systems, the leader may have to take "yes-no" decisions (Wen and Yang 1990). This type of decisions can be described by the introduction of binary variables in the model. Assuming that the optimisation variables are separable and appear in linear relations, the following mixed integer BLPP is derived (Shimizu et al. 1997): 


$$
\begin{array}{ll} 
& \min _{x_{1}, x_{2}, y_{1}, y_{2}} F\left(x_{1}, x_{2}, y_{1}, y_{2}\right)=L_{1}+L_{2}^{T} x_{1}+L_{3}^{T} y_{1}+L_{4}^{T} x_{2}+L_{5}^{T} y_{2}, \\
\text { s.t. } & G_{1} x_{1}+G_{2} y_{1}+G_{3} x_{2}+G_{4} y_{2}+G_{5} \leq 0, \\
& \min _{y_{1}, y_{2}} f\left(x_{1}, x_{2}, y_{1}, y_{2}\right)=l_{1}+l_{2}^{T} x_{1}+l_{3}^{T} y_{1}+l_{4}^{T} x_{2}+l_{5}^{T} y_{2}, \\
& \text { s.t. } g_{1} x_{1}+g_{2} y_{1}+g_{3} x_{2}+g_{4} y_{2}+g_{5} \leq 0,
\end{array}
$$

where $x_{1}, x_{2}, y_{1}$ and $y_{2}$ are the optimisation variables, $x_{1} \in X_{1} \subseteq \mathbb{R}^{n x_{1}}, x_{2} \in\{0,1\}^{n x_{2}}$ $y_{1} \in Y_{1} \subseteq \mathbb{R}^{n y_{1}}, y_{2} \in\{0,1\}^{n y_{2}} \cdot\left[L_{2}\right]_{n x_{1}},\left[L_{3}\right]_{n y_{1}},\left[L_{4}\right]_{n x_{2}},\left[L_{5}\right]_{n y_{2}},\left[l_{2}\right]_{n x_{1}},\left[l_{3}\right]_{n y_{1}},\left[l_{4}\right]_{n x_{2}}$ $\left[l_{5}\right]_{n y_{2}}$ are vectors defined in the real space. The matrices $\left[G_{1}\right]_{n u \times n x_{1}}$, $\left[G_{2}\right]_{n u \times n y_{1}}$ $\left[G_{3}\right]_{n u \times n x_{2}}, \quad\left[G_{4}\right]_{n u \times n y_{2}}, \quad\left[G_{5}\right]_{n u \times 1}, \quad\left[g_{1}\right]_{n u \times n x_{1}}, \quad\left[g_{2}\right]_{n u \times n y_{1}},\left[g_{3}\right]_{n u \times n x_{2}},\left[g_{4}\right]_{n u \times n y_{2}}$ $\left[g_{5}\right]_{n u \times 1}$ correspond to the constraints, also defined in the real space.

If the integrality conditions, with respect to $x_{2}$, are moved to the upper level, a multi-parametric mixed integer linear programming (mp-MILP), with $x_{1}$ and $x_{2}$ being the parameters, can be formulated as follows (Formulation 23):

$$
\begin{aligned}
& \quad \min _{y_{1}, y_{2}} f\left(x, y_{1}, y_{2}\right)=l_{1}^{\prime}+l_{2}^{T} x+l_{3}^{T} y_{1}+l_{5}^{T} y_{2}, \\
& \text { s.t. } g_{2}^{\prime} y_{1}+g_{4}^{\prime} y_{2} \leq g_{5}^{\prime}+g_{1}^{\prime} x,
\end{aligned}
$$

where: $x=\left[x_{1} \mid x_{2}\right]^{T} ; l_{1}^{\prime}=l_{1} ; l_{2}^{\prime}=\left[l_{2} \mid l_{4}\right]^{T} ; l_{3}^{\prime}=l_{3} ; l_{5}^{\prime}=l_{5} ; g_{1}^{\prime}=-\left[g_{1} \mid g_{3}\right]^{T} ; g_{2}^{\prime}=g_{2}$; $g_{4}^{\prime}=g_{4} ; g_{5}^{\prime}=-g_{5}$.

The mp-MILP problem in (23) can be solved applying the algorithm of Dua and Pistikopoulos (2000), from which the following group of $K$ solutions are obtained:

$$
\begin{aligned}
& y_{2}^{k}=\bar{y}_{2}^{k}, \\
& y_{1}^{k}=m^{k}+n^{k} x \Leftrightarrow y_{1}^{k}=m^{k}+n_{1}^{k} x_{1}+n_{2}^{k} x_{2}, \quad k=1,2, \ldots, K, \\
& H^{k} x \leq h^{k} \Leftrightarrow H_{1}^{k} x_{1}+H_{2}^{k} x_{2} \leq h^{k} .
\end{aligned}
$$

Introducing these expressions in (22), a set of $K$ independent MILPs is obtained:

$$
\begin{aligned}
& F\left(x_{1}, x_{2}\right)=\min _{x_{1}, x_{2}}\left\{L_{1}^{\prime k}+L_{2}^{\prime k^{T}} x_{1}+L_{4}^{\prime k^{T}} x_{2}\right\}, \\
& \text { s.t. } \quad G_{1}^{\prime k} x_{1}+G_{3}^{\prime k} x_{2} \leq G_{5}^{\prime k}
\end{aligned}
$$

with: $L_{1}^{\prime k}=L_{1}+L_{3} m^{k}+L_{5} \bar{y}_{2}^{k} ; L_{2}^{\prime k}=L_{2}+L_{3} n_{1}^{k} ; L_{4}^{\prime k}=L_{4}+L_{3} n_{2}^{k}$;

$$
\begin{aligned}
& G_{1}^{\prime}=G_{1}+G_{2} n_{1}^{k}, \\
& G_{3}^{\prime}=G_{3}+G_{2} n_{2}^{k}, \\
& G_{5}^{\prime}=-\left(G_{4} \bar{y}_{2}^{k}+G_{5}+G_{2} m^{k}\right), \\
& G_{1}^{\prime k}=\left[G_{1}^{\prime} \mid H_{1}^{k}\right]^{T} ; G_{3}^{\prime k}=\left[G_{3}^{\prime} \mid H_{2}^{k}\right]^{T} ; G_{5}^{\prime k}=\left[G_{3}^{\prime} \mid h^{k}\right]^{T} .
\end{aligned}
$$

The solution of the $K$ MILPs in (25) results in the selection of the global optimum by direct comparison. 
Table 6 Solution for Problem (26)

$$
\begin{aligned}
& F=-1011.67 \\
& f=-4673.34 \\
& y_{1}=0.00 \\
& y_{2}=75.00 \\
& y_{3}=21.67 \\
& x_{1}=0 \\
& x_{2}=1 \\
& x_{3}=0 \\
& x_{4}=1
\end{aligned}
$$

The proposed strategy will be illustrated by the following MILP|LP BLPP introduced by (Wen and Yang 1990):

$$
\begin{array}{rl}
\min _{x, y} & F(x, y)=-\left(20 x_{1}+60 x_{2}+30 x_{3}+50 x_{4}+15 y_{1}+10 y_{2}+7 y_{3}\right), \\
\text { s.t. } \min _{y} & f(x, y)=-\left(20 y_{1}+60 y_{2}+8 y_{3}\right), \\
\text { s.t. } & 5 x_{1}+10 x_{2}+30 x_{3}+5 x_{4}+8 y_{1}+2 y_{2}+3 y_{3} \leq 230, \\
& 20 x_{1}+5 x_{2}+10 x_{3}+10 x_{4}+4 y_{1}+3 y_{2} \leq 240, \\
& 5 x_{1}+5 x_{2}+10 x_{3}+5 x_{4}+2 y_{1}+y_{3} \leq 90, \\
& x \in\{0,1\}, \\
& y \geq 0 .
\end{array}
$$

Moving the integrality constraint to the outer level, the inner problem can be rewritten as a mp-LP with $x$ being the parameter. Its solution results in the following parametric expressions (single critical region):

$$
\begin{aligned}
& y_{1}=0 \\
& y_{2}=-6.667 x_{1}-1.667 x_{2}-3.333 x_{3}-3.333 x_{4}+80, \\
& y_{3}=2.778 x_{1}-2.222 x_{2}-7.778 x_{3}+0.5556 x_{4}+23.33 \\
& 0 \leq x_{1}, x_{2}, x_{3}, x_{4} \leq 1 .
\end{aligned}
$$

Introducing these expressions in the leader's problem, and taking into account the binary nature of $x$, the following MILP problem is obtained:

$$
\begin{array}{ll}
\min _{x} & F=-\left(20 x_{1}+60 x_{2}+30 x_{3}+50 x_{4}+15 y_{1}+10 y_{2}+7 y_{3}\right), \\
\text { s.t. } & y_{1}=0, \\
& y_{2}=-6.667 x_{1}-1.667 x_{2}-3.333 x_{3}-3.333 x_{4}+80.00, \\
& y_{3}=2.778 x_{1}-2.222 x_{2}-7.778 x_{3}+0.5556 x_{4}+23.33, \\
& x \in\{0,1\}, \\
& y \geq 0 .
\end{array}
$$

Table 6 presents the solution for Problem (28), and subsequently for Problem (26). The result obtained is identical to the one obtained by Wen and Yang (1990).

\section{Concluding remarks}

We have described the foundations of a novel global optimisation strategy for the solution of general classes of bilevel programming based on our recent developments in multi-parametric programming. It was shown that bilevel linear, quadratic and mixed-integer linear programs, also involving uncertainty, can be effectively solved. It 
was further shown that issues related to global optimality for both levels of the bilevel program can be addressed.

The proposed approach can be further extended for trilevel and general multilevel programming problems (Ruan et al. 2004), Stackelberg-Nash Equilibrium type of problems (Liu 1998), as well as to diverse application areas, such as hierarchical control structures (Stephanopoulos and $\mathrm{Ng}$ 2000) and enterprise-wide supply chain optimisation problems (Cao and Chen 2006). These are currently undergoing further investigation.

Acknowledgements Financial support from EPSRC (GR/T02560/01) and Marie Curie European Project PRISM (MRTN-CT-2004-512233) is gratefully acknowledged.

\section{Appendix A: the mp-QP algorithm}

The steps of the solution algorithm (Dua et al. 2002) for mp-QP problems are as follows:

Step 1 Treating $\theta$ as a free variable, solve the following mp-QP problem to obtain a feasible point $\left[\theta_{Q}\right]$ :

$$
\begin{array}{ll} 
& z(\theta)=\min _{x} c^{T} x+\frac{1}{2} x^{T} Q x, \\
\text { s.t. } & A x \leq b+F \theta, \\
& x \in X \subseteq \mathbb{R}^{n} \\
& \theta \in \Theta \subseteq \mathbb{R}^{m} .
\end{array}
$$

where $c$ is a constant vector of dimension $n, Q$ is an $(n \times n)$ symmetric positive definite constant matrix, $A$ is a $(p \times n)$ constant matrix, $F$ is a $(p \times m)$ constant matrix, $b$ is a constant vector of dimension $p$ and $X$ and $\Theta$ are compact polyhedral convex sets of dimensions $n$ and $m$, respectively.

Step 2 Fix $\theta=\theta_{Q}$ and solve (29) to obtain $\left[x\left(\theta_{Q}\right), \lambda\left(\theta_{Q}\right)\right]$, where $\lambda$ are the Lagrange multipliers.

Step 3 Applying the Basic Sensitivity Theorem (Fiacco 1976) to (29), and considering first-order estimations, the following expressions are obtained:

$$
\left[\begin{array}{c}
x_{Q}(\theta) \\
\lambda_{Q}(\theta)
\end{array}\right]=-\left(M_{Q}\right)^{-1} N_{Q}\left(\theta-\theta_{Q}\right)+\left[\begin{array}{c}
x\left(\theta_{Q}\right) \\
\lambda\left(\theta_{Q}\right)
\end{array}\right],
$$

where,

$$
\begin{gathered}
M_{Q}=\left(\begin{array}{cccc}
Q & A_{1}^{T} & \cdots & A_{p}^{T} \\
-\lambda_{1} A_{1} & -V_{1} & & \\
\vdots & & \ddots & \\
-\lambda_{p} A_{p} & & & -V_{p}
\end{array}\right), \\
N_{Q}=\left(Y, \lambda_{1} F_{1 .}, \ldots, \lambda_{p} F_{p .}\right)^{T}
\end{gathered}
$$

$V_{i}=A_{i .} x\left(\theta_{Q}\right)-b_{i}-F_{i .} \theta_{Q}$ and $Y$ is a null matrix of dimension $(n \times m)$. Obtain $\left[x_{Q}(\theta), \lambda_{Q}(\theta)\right]$ from (30).

Step 4 Form a set of inequalities, $C R^{R}$ :

$$
C R^{R}=\left\{\breve{A} x_{Q}(\theta) \leq \breve{b}+\breve{F} \theta, \tilde{\lambda}_{Q}(\theta) \geq 0, C R^{I G}\right\},
$$


where $\breve{A}, \breve{b}$ and $\breve{F}$ correspond to the inactive inequalities, $\tilde{\lambda}_{Q}$ to the active constraints and $\mathrm{CR}^{\mathrm{IG}}$ represents a set of linear inequalities defining an initial given region. From the parametric inequalities thus obtained, the redundant inequalities are removed and a compact representation of the $\mathrm{CR}^{Q}$ is obtained: $C R^{Q}=\Delta\left\{C R^{R}\right\}$, where $\Delta$ is an operator which removes redundant constraints - for a procedure to identify redundant constraints (see Gal 1995).

Step 5 Define the rest of the region, $\mathrm{CR}^{\text {rest }}=\mathrm{CR}^{\mathrm{IG}}-\mathrm{CR}^{Q}$.

Step 8 If there are no more regions to explore, go to next step, otherwise go to Step 1.

Step 9 Collect all the solutions and unify the regions having the same solution to obtain a compact representation.

\section{References}

Acevedo, J., Pistikopoulos, E.N.: A multiparametric programming approach for linear process engineering problems under uncertainty. Ind. Eng. Chem. Res. 36, 717-728 (1997)

Aiyoshi, E., Shimizu, K.: Hierarchical decentralized systems and its new solution by a barrier method. IEEE Trans. Syst. Man Cybern. 11(6), 444-449 (1981)

Başar, T., Olsder, G.J.: Dynamic Noncooperative Game Theory. Academic Press, London (1982)

Bard, J.F., Falk, J.: An explicit solution to the multi-level programming problem. Comput. Oper. Res. 9, 77-100 (1982)

Cao, D., Chen, M.: Capacitated plant selection in a decentralized manufacturing environment: a bilevel optimization approach. Eur. J. Oper. Res. 169(1), 97-110 (2006)

Clark, P.A.: Bilevel programming for steady-state chemical process design - II. performance study for nondegenerate problems. Comput. Chem. Eng. 14(1), 99-109 (1990)

Clark, P.A., Westerberg, A.W.: Bilevel programming for steady-state chemical process design - I. fundamentals and algorithms. Comput. Chem. Eng. 14(1), 87-97 (1990)

Dempe, S.: Annotated bibliography on bilevel programming and mathematical programs with equilibrium constraints. Optim. 52(3), 33-359 (2003)

Dempe, S., Kalashnikov, V., Ríos-Mercado, R.Z.: Discrete bilevel programming: Application to a natural gas cash-out problem. Eur. J. Oper. Res. 166, 469-488 (2005)

Deng, X.: Complexity issues in bilevel linear programming. In: Pardalos, P.M., Varbrand, P., Migdalas, A. (eds.) Multilevel Optimization: Algorithms and Applications, pp. 149-164. Kluwer Academic Publishers. Dordrecht (1998)

Dua, V.: Parametric programming techniques for process engineering problems under uncertainty. $\mathrm{PhD}$ thesis. Department of Chemical Engineering and Chemical Technology Imperial College of Science, Technology and Medicine London, UK (2000)

Dua, V., Pistikopoulos, E.N.: An algorithm for the solution of multiparametric mixed integer linear programming problems. Ann. Oper. Res. 99, 123-139 (2000)

Dua, V., Bozinis, A., Pistikopoulos, E.N.: A multiparametric programming approach for mixed-integer quadratic engineering problems. Comput. Chem. Eng. 26, 715-733 (2002)

Evans, G.W.: An overview of thecniques for solving multiobjective mathematical programs. Manage. Sci. 30(11), 1268-1282 (1984)

Fiacco, A.V.: Sensitivity analysis for nonlinear programming using penalty methods. Math. Program. 10, 287-311 (1976)

Fiacco, A.V.: Introduction to Sensitivity and Stability Analysis in Nonlinear Programming. Academic Press, New York (1983)

Floudas, C.A.: Deterministic Global Optimization. Kluwer Academic Publishers, Dordrecht, (2000)

Floudas, C.A., Pardalos, P.M., Adjiman, C.S., Esposito, W.R., Gümüs, Z.H., Harding, S.T., Klepeis, J.L., Meyer, C.A., Schweiger, C.A.: Handbook of Test Problems in Local and Global Optimization. Kluwer Academic Publisher, Dordrecht (1999)

Fortuny-Amat, J., McCarl, B.: A representation and economic interpretation of a two-level programming problem. J Oper. Res. Soc. 32(9), 783-792 (1981)

Gal, T.: Postoptimal Analyses, Parametric Programming, and Related Topics. de Gruyter, New York, (1995)

Hansen, P., Jaumard, B., Savard, G.: New brach-and-bound rules for linear bilevel programming. SIAM J. Sci. Stat. Comput. 13, 1194-1217 (1992) 
Lai, Y.: Hierarchical optimization: a satisfactory solution. Fuzzy Sets Syst. 77, 321-335 (1996)

LeBlanc, L.J., Boyce, D.E.: A bilevel programming algorithm for exact solution of network design problemwith user-optimal flows. Trans. Res. - Part B Meth. 20, 259-265 (1985)

Liu, B.: Stakelberg-nash equilibrium for multilevel programming with multiple followers using genetic algorithms. Comput. Math. Appl. 36(7), 79-89 (1998)

Muu, L.D., Quy, N.V.: A global optimization method for solving convex quadratic bilevel pogramming problems. J. Global Optim. 26, 199-219 (2003)

Ruan, G.Z., Wang, S.Y., Yamakamoto, Y., Zhu, S.S.: Optimality conditions and geometric properties of linear multilevel programming problem with dominated objective functions. J Optim. Theory Appl. 123(2), 409-429 (2004)

Ryu, J., Dua, V., Pistikopoulos, E.N.: A bilevel programming framework for enterprise-wide process networks under uncertainty. Comput. Chem. Eng. 28, 1121-1129 (2004)

Sakizlis, V., Perkins, J.D., Pistikopoulos, E.N.: Parametric controllers in simultaneous process and control design optimization. Ind. Eng. Chem. Res. 42, 4545-4563 (2003)

Sakizlis, V., Perkins, J.D., Pistikopoulos, E.N.: Recent advances in optimization-based simulataneous process and control design. Comput. Chem. Eng. 28(10), 2069-2086 (2004 a)

Sakizlis, V., Kakalis, M.P., Dua, V., Perkins, J.D., Pistikopoulos, E.N.: Design of robust controllers via parametric programming. Automatica 40, 189-201 (2004 b)

Shih, H., Lai, Y., Lee, E.S.: Fuzzy approach for multi-level programming problems. Comput. Oper. Res. 23(1), 73-91 (1996)

Shimizu, K., Ishizuka, Y., Bard, J.F.: Nondifferentiable and Two-Level Mathematical Programming. Kluwer Academic Press, Boston (1997)

Stephanopoulos, G., Ng, C.: Perspectives on the synthesis of plant-wide control structures. J. Process Control 10, 97-111 (2000)

Tabucanon, M.T.: Multiple Criteria Decision Making in Industry. Elsevier, Amsterdam (1988)

Vicente, L.N., Calamai, P.H.: Bilevel and multilevel programming: A bibliography review. J. Global Optim. 5(3), 291-306 (1994a)

Vicente, L.N., Savard, G., Júdice, J.: Descent approaches for quadratic bilevel programming. J. Optim. Theory Appl. 81, 379-399 (1994b)

Vicente, L.: Bilevel programming. Master's thesis. Department of Mathematics, University of Coimbra, Coimbra (1992)

Visweswaran, V., Floudas, M.G., Ierapetritou, C.A., Pistikopoulos, E.N.: A decomposition-based global optimization approach for solving bilevel linear and quadratic programs. In: Floudas, C.A., Pardalos, P.M. (eds.) State of the Art in Global Optimization, pp. 139-162. Kluwer Academic Publishers, Dordrecht (1996)

Wen, U.P., Yang, Y.H.: Algorithms for solving the mixed integer two-level linear programming problem. Comput. Oper. Res. 17(2), 133-142 (1990) 